\title{
Lithium Ion SuperPolymer@ High-Performance Battery for Ultra-Safe, Long-Range ZEVs, HEVs, and PHEVs
}

\begin{abstract}
Sankar Dasgupta*
Electrovaya's Lithium Ion SuperPolymer ${ }^{\circledR}$ based battery is a cost-effective solution for long-range, ultrasafe zero-emission (battery electric) and low-emission (plug-in hybrid) vehicles. Optimized for transportation, Electrovaya has developed fully integrated power system solutions with large-format cells, large-format modules, and integrated an intelligent battery management system for safe and effective scale-up. Electrovaya's proprietary SuperPolymer ${ }^{\circledR}$ technology is independent of the composition of the positive electrode active material and so multiple chemistry solutions are available, including the MN-Series, a Lithiated Manganese Oxide based system, with up to 50\% higher energy density and comparable safety characteristics to Electrovaya's Phosphate-Series solution.
\end{abstract}

This paper details the design approach and recent test results of Electrovaya's solutions for (1) plug-in hybrids, with its program with New York State on a Ford Escape platform; (2) passenger vehicles, as demonstrated in a 30kWh ZEV in Norway; (3) fleet vehicles, such as an 80kWh delivery vehicle in partnership with Unicell, Purolator and the Canadian Government; and (4) off-road vehicles, such as its project with New York State Parks.

Electrovaya's scalable power train solution is easily tailored to the demands of passenger, fleet, off-road, and heavy-duty applications. This solution is cost-competitive to ICE-based vehicles and superior in performance, safety and operating cost. Electrovaya's SuperPolymer ${ }^{\circledR}$ technology gives superior safety and performance and is exceptionally suited to large format systems necessary for transportation applications.

\section{INTRODUCTION}

Electrovaya, particularly over the past year, has made great strides in its development of integrated battery systems for electric drive applications. These systems are designed at each stage - chemistry, cell, module, system with integrated intelligent electronics - for scale-up, safety, performance, and cost-effectiveness.

This paper details several of Electrovaya's advancements and experience with plug-in hybrid electric vehicles and full battery electric vehicles. For further details and up-to-date testing results please do not hesitate to contact Electrovaya directly.

\section{INTEGRATED SYSTEM APPROACH}

Electrovaya's philosophy is holistic to the entire

* Electrovaya Inc., 2645 Royal Windsor Dr, Mississauga ON L5J 1K9, Canada

Phone: 905-855-4610, Fax: 905-822-7953

sdasgupta@electrovaya.com

www.electrovaya.com battery system. In other words, Electrovaya approaches the design of its battery systems for electric drive application with the whole system in mind.

In fact, every element (mechanical, thermal, electrochemical, electrical, electronic) is optimized at every stage (chemistry choice, cell design, module design, system design, electronics design). The optimization parameters demand scale-up, performance, safety, and cost competitiveness.

As a result, Electrovaya's battery systems are uniquely optimized for electric drive applications.

\subsection{System Level o: Platform Independence $\rightarrow$ Multiple Chemistry Options}

Electrovaya's polymer battery is a platform, independent of electrode materials. Hence, Electrovaya can incorporate the advances in material and electrode chemistry as they appear. This protects its technology from being a "transitional" solution. 
Currently, Electrovaya offers three chemistry solutions:

1. Cobaltate $(150-220 \mathrm{Wh} / \mathrm{kg})$ - small systems, i.e. cells up to $10 \mathrm{Ah}$

2. Phosphate (110-130 Wh/kg) - a lithium iron phosphate electrode material for large systems, including transportation

3. MN-Series (170-210 Wh/kg) - a proprietary material that offers both superior energy density (energy density up to $50 \%$ better than phosphate) and safety (comparable to phosphate)

For transportation applications, Electrovaya prefers the MN Series as it gives more than $50 \%$ higher energy density than phosphate with comparable safety and rate properties.

\subsection{System Level 1: Cell-Level Advantages}

\subsubsection{SUPERPOLYMER®: SUPERIOR ENERGY DENSITY}

Electrovaya's technology is typically characterized by higher energy densities (gravimetrically and volumetrically) than comparable competitors. This competitive advantage is due to Electrovaya's proprietary nanostructured electrolyte embedded throughout the structure, such that lithium mobility is very high (multiple patents) and provide multiple transport routes. The "SuperPolymer" name of Electrovaya's proprietary Lithium Ion SuperPolymer ${ }^{\circledR}$ refers to this proprietary advantage and technology differentiation.

Electrovaya has nearly 180 patents issued and pending globally, including about 30 in the US. The technology has won many awards from NASA, Frost \& Sullivan and others, while Deloitte has listed Electrovaya as one of the fastest growing companies in North America.

\subsubsection{BALANCED ENERGY-POWER CELL OPTIMIZATION AVAILABLE}

Lithium batteries have typically been optimized for either (1) consumer electronics, which demand high-energy density and low-rate discharge; or (2) hybrid vehicles, which demand high-rate discharges. For electric drive applications neither of the first optimization, high-energy/low-power or the second, high-power/low-energy optimization is optimal.

Electrovaya is pleased to offer a balanced energy/ power optimization. This provides both the superior energy density and high rate battery solution required for plug-in hybrids or full electric vehicles.
The balanced-optimization offers:

o Superior energy density of $170 \mathrm{Wh} / \mathrm{kg}-210 \mathrm{Wh} /$ $\mathrm{kg}$

o High volumetric energy

- Cell level of 300 - $450 \mathrm{Wh} / \mathrm{liter}$

- System level - an additional 22\% improvement in volumetric energy is gained due to prismatic format (as compared to cylindrical cells)

o High-rate capability of up to $10 \mathrm{C}$ pulses, which is adequate for most applications

\subsection{System Level 2: Integrated Intelligence}

Electrovaya's battery systems offer integrated, intelligent battery management system (BMS). This CANbus-enabled solution provides the following functions:

o Multi-level safety

o Battery system equalization - extends life, reliability, and performance

o Protection against overcharge, over temperature, over discharge, over current and more

o State of charge and state of health: accurate calculations with intelligence \& self-learning

o Information for the driver display and alarms

o For further details on Electrovaya's proprietary BMS, please contact us directly

\subsection{System Level 3: Scale-up \& Cell Geometry}

Scale-up capability is key to the reliability of large battery systems. Also critical is the form factor for thermal and scale-up performance. Whereas most batteries are manufactured spiral-wound cylindrical cells Electrovaya manufactures large-format prismatic (flat) cells.

In numbers, the importance of scale-up is quickly evident. Electrovaya's standard 1.5kWh module would require 356 (phosphate) standard "18650" cells or 166 (cobalt) cells. For a $25 \mathrm{kWh}$ battery only 17 Electrovaya modules would be needed. In contrast, this same system would require 2768 (commercial cobalt) cells or 5932 (commercial phosphate) cells. In addition, there is a $22 \%$ volumetric energy density loss with cylindrical cells due to their geometry.

Spiral wound cylindrical batteries are poor choices for large systems and have been repeatedly tried with often challenging results in large systems. Their ubiquity is due to high production in China and East Asia. Problems include:

o Thermal issues: heat removal is poor and so hot spots internally can cause poor performance and significant thermal problems 
o Packaging: the cell is contained in a metal canister, which when under pressure can explode. The metal canister can then become metal shrapnel

o Scale-up limitations: These small cells entail:

- $22 \%$ volumetric loss in energy density

- Multiple interconnects and many points of failure

- Highly complex integration

- Complex electronic management

In contrast, Electrovaya's large-format prismatic technology has important implications:

o Prismatic cells are much safer than spiral wound cells due to their packaging. Under stress a prismatic pouched cell will "puff," much like a microwaveable bag of popcorn will pop when heated. In comparison, a cylindrical wound cell is contained in a metal "can." As a result, Electrovaya's standard cells cannot explode, because there is no mechanism that could cause compression of gases inside the cell.

o Excellent thermal properties and optimized thermal management

o Large format cells offer significant scale-up advantages

o Very good reliability. Electrovaya's technology has been tested for low-temperature performance, vibration, reliability and 10-year calendar life in various applications.

\subsection{System Level 4: Thermal Optimization}

Thermal characteristics of large lithium ion battery packs have been a major problem area for system designers and integrators, with respect to their cycle life, energy density, and performance. Active thermal control through the use of liquid or air-cooling is ubiquitous. Cylindrical cells are especially prone to failure due to its difficult thermal characteristics and poor heat removal from inside the cell. This has been a major technical challenge to cylindrical cell technology and one of the reasons why the cells cannot be scaled up to larger sizes as required by the vehicle community.

Electrovaya's prismatic cells are designed similar to a plate and frame heat exchanger and all or most of the heat produced can be efficiently removed through the terminals. This design effectively means that Electrovaya can produce cells from 3Ah to 100Ah or even 200Ah.

\section{JIT AVAILABILITY}

Electrovaya has its main manufacturing base in North America. Additional plants will shortly be online across Europe and Asia. Plants are planned for South America. Electrovaya is able to support is partners with just-in-time (JIT) production for cells, modules, or complete systems.

\section{PLUG-IN HYBRID ELECTRIC VEHICLE EXPERIENCE}

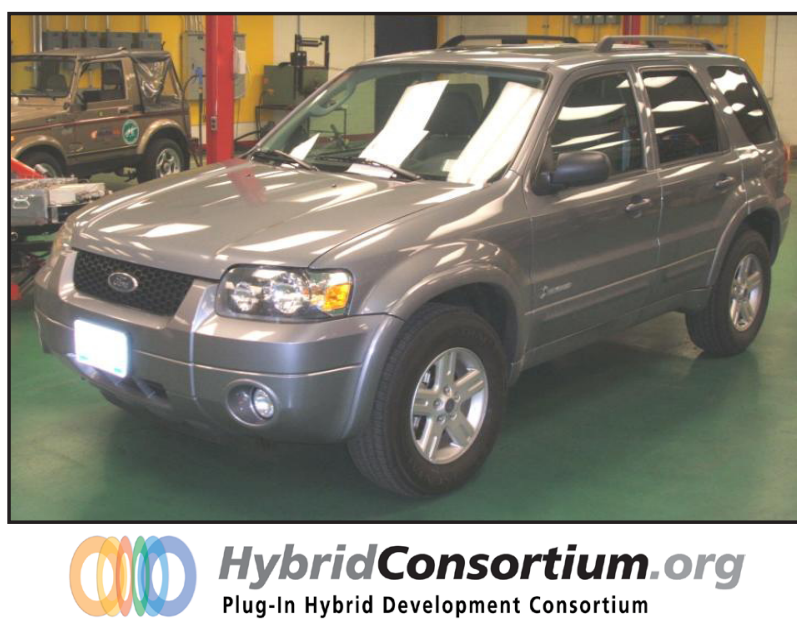

Figure 1: Ford Escape PHEV, delivered to the state of

New York in August 2007

Electrovaya is a cofounder of the Plug-In Hybrid Consortium

Electrovaya recently delivered its first Ford Escape plug-in hybrid (Figure 1). Since the Electrovaya cells are more efficient volumetrically than the phosphate cells, the energy capacity of the battery was doubled to nearly $15 \mathrm{kWh}$. This provided a capability of this SUV to reach 130 miles per gallon.

\section{BATTERY ELECTRIC VEHICLE EXPERIENCE}

This (Figure 2) electric vehicle used the Smart car chassis, where the space available for a battery was very small. Electrovaya managed to install nearly

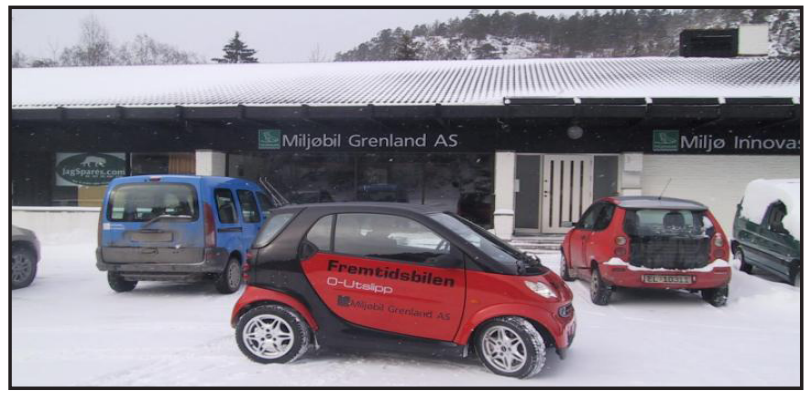

Figure 2: 3okWh passenger vehicle with partner, Miljobil Grenland (Norway) 
$30 \mathrm{kWh}$ of energy and the vehicle has been operating through the Norwegian winter, the battery providing all the energy requirements including motive force as well as heating the inside of the vehicle.

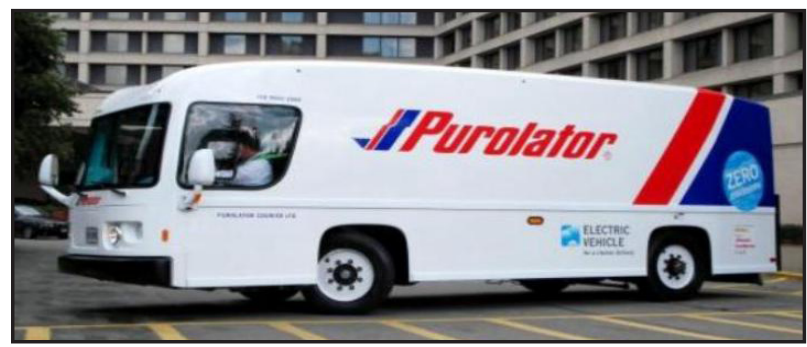

Figure 3: 80-10okWh fleet vehicle

The market for a courier delivery van (Figure 3) is large and an excellent early user for zero emission electric vehicles. The delivery vans are used in urban settings and are in a frequent stop-go mode. The typical range for these vehicles is about $120 \mathrm{~km}$ (70 miles).

Electrovaya has designed a modular system, where the energy necessary can be matched with the modules. Initially this delivery van will have about $110 \mathrm{kWh}$ of energy to provide proof of concept. In actual production, it is expected that a $60 \mathrm{kWh}$ pack will be able to meet the range and power requirements for this class of vehicles. The electric motor operates at a nominal 600Volts and Unicell and Arvin Meritor are the designers of the vehicle and chassis, respectively.

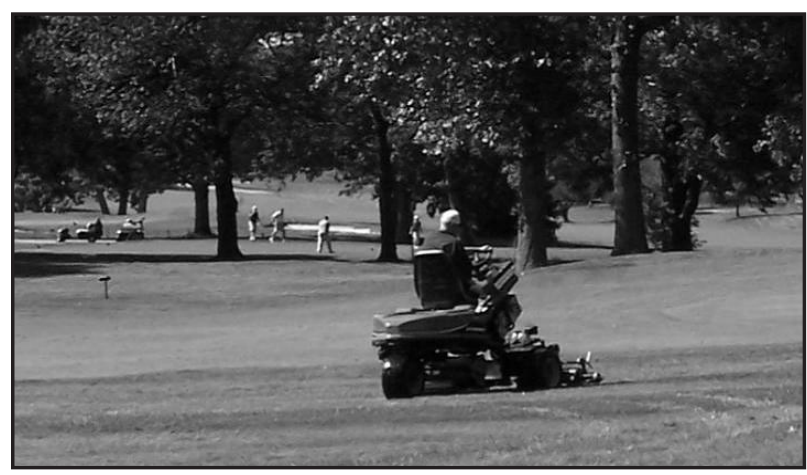

Figure 4: Off-road vehicle

Electrovaya is involved in a number of demonstration projects for off-road vehicles (Figure 4). Here again, the drivers are lower maintenance, clean transportation, zero emission, as well as quiet in mode of operation.

The Maya-100 has up to $300 \mathrm{~km}$ range with highway speeds (Figure 5). This was one of the first full electric SUVs built by Electrovaya using a chassis manufactured by CAMI, a vehicle producer in Ontario, Canada. This

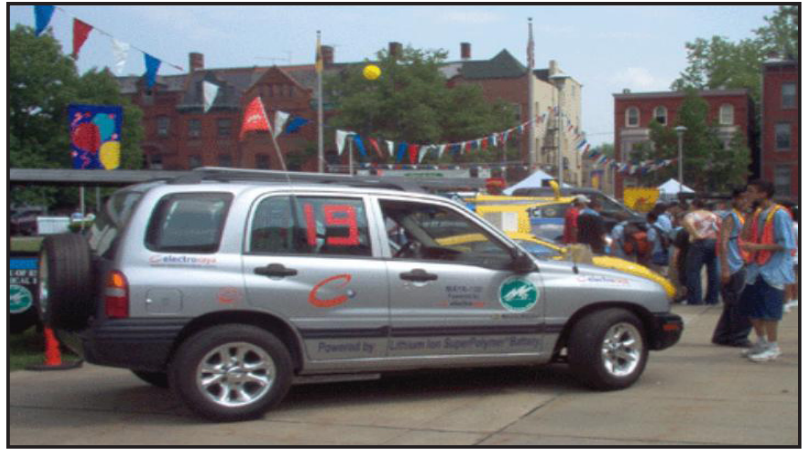

Figure 5: Maya-100 passenger vehicle

vehicle operates a motor at $150 \mathrm{~V}$ and is powered by Electrovaya's 40kWh SuperPolymer ${ }^{\circledR}$ battery system. The vehicle has been used continuously for the last three years.

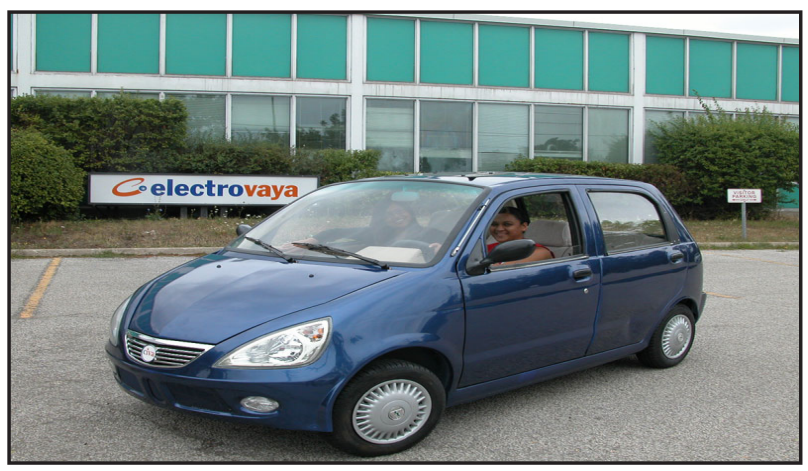

Electrovaya is supplying modular battery packs for the NEV market and is working with some suppliers. This NEV, or low-speed vehicle market, is a niche market in North America and Europe and may be a good transportation segment for early adopters to zero emission vehicles.

The typical NEV battery pack from Electrovaya is a $10 \mathrm{kWh}$ pack with a standalone battery management system and an integrated charger. NEV integrators can take the Electrovaya power system and integrate in a "plug and play" mode (Figure 6). 